cond-mat/9508056

\title{
Quantum transport in disordered wires: Equivalence of one-dimensional $\sigma$ model and Dorokhov-Mello-Pereyra-Kumar equation
}

\author{
P. W. Brouwer \\ Instituut-Lorentz, University of Leiden, P.O. Box 9506, 2300 RA Leiden, The Netherlands \\ K. Frahm \\ Service de Physique de l'État Condensé, Commissariat à l'Energie Atomique Saclay, 91191 \\ Gif-sur-Yvette, France
}

(August 15, 1995)

\begin{abstract}
The two known non-perturbative theories of localization in disordered wires, the Fokker-Planck approach due to Dorokhov, Mello, Pereyra, and Kumar, and the field-theoretic approach due to Efetov and Larkin, are shown to be equivalent for all symmetry classes. The equivalence had been questioned as a result of field-theoretic calculations of the average conductance by Zirnbauer [PRL 69, 1584 (1992)], which disagreed with the Fokker-Planck approach in the symplectic symmetry class. We resolve this controversy by pointing to an incorrect implementation of Kramers degeneracy in these calculations, and we derive modified expressions for the first two conductance moments which agree well with existing numerical simulations from the metallic into the localized regime.
\end{abstract}

PACS numbers: 72.15.Rn, 72.10.Bg, 02.50.-r 


\section{INTRODUCTION}

There are two known approaches to the theory of phase-coherent conduction and localization in disordered wires: The first is the Fokker-Planck approach of Dorokhov, Mello, Pereyra, and Kumar. 0 The second is the field-theoretic approach of Efetov and Larkin, which leads to a supersymmetric nonlinear $\sigma$ model. 6 - Both approaches provide a description of quantum transport that is independent of microscopic details of the disordered wire. The only properties which enter are its length $L$, the elastic mean free path $\ell$, the number $N$ of propagating transverse modes at the Fermi level (referred to as "channels"), and the symmetry index $\beta \in\{1,2,4\}$ (depending on the presence or absence of time-reversal and/or spin-rotational symmetry). In the first approach, the transfer matrix is expressed as a product of a large number of random matrices. As more matrices are added to this product, the transmission eigenvalues $T_{n}$ execute a Brownian motion. (The $T_{n}$ are the $N$ eigenvalues of the transmission matrix product $t^{\dagger} t$.) The resulting Fokker-Planck equation for the $L$-dependence of the distribution $P\left(T_{1}, \ldots, T_{N}\right)$ is known as the Dorokhov-MelloPereyra-Kumar (DMPK) equation. In the second approach, one starts from the random Hamiltonian of the disordered wire and then expresses averages of Green's functions 6 . moments of the transmission eigenvalues 811 as integrals over matrices $Q$ containing both commuting and anticommuting variables. These so-called supermatrices are restricted by the nonlinear constraint $Q^{2}=1$ and give rise to a field theory known as the one-dimensional nonlinear $\sigma$ model.

In the last decade, research on the Fokker-Planck and field-theoretic approach has proceeded quite independently. Recently, exact results for the average conductance $\langle G\rangle$, its variance $\operatorname{var} G$, and the density $\rho(T)=\left\langle\sum_{n} \delta\left(T-T_{n}\right)\right\rangle$ of transmission eigenvalues were obtained from both approaches. For the unitary symmetry class (no time-reversal symmetry; $\beta=2$ ), the DMPK equation was solved exactly by Beenakker and Rejaei.12 The construction of a set of biorthogonal polynomials for this exact solution then allowed for the exact computation of $\langle G\rangle$, $\operatorname{var} G$, and $\rho(T)$ for arbitrary $N$ and $L$ in the case $\beta=2.13$ Although there exists a formal solution for the other two symmetry classes [orthogonal class (time-reversal symmetry without spin-orbit scattering: $\beta=1$ ) and symplectic class (timereversal symmetry with spin-orbit scattering; $\beta=4)$ ], $\$ 4$ no exact results for $\langle G\rangle$, var $G$, and $\rho(T)$ have been obtained. Concerning the $\sigma$ model, an important and substantial progress was the development of "super Fourier analysis" by Zirnbauer.10 This allowed the exact calculation 10,11 of $\langle G\rangle$ and $\operatorname{var} G$ for all $\beta$ in the thick-wire limit $N \rightarrow \infty, L / \ell \rightarrow \infty$ at fixed ratio $N \ell / L$. The eigenvalue density $\rho(T)$ was computed from the $\sigma$ model by Rejaei, 1 in the thick-wire limit and for the case $\beta=2$.

If one takes the thick-wire limit of the $\beta=2$ results for $\langle G\rangle, \operatorname{var} G$, and $\rho(T)$ from the DMPK equation, they agree precisely with those from the $\sigma$ model. 13,15 For $\beta=1$ and 4 , a comparison of the two approaches has only been possible in the metallic regime $\ell \ll L \ll N \ell$. where the results for $\langle G\rangle$ and $\operatorname{var} G$ from the DMPK equation 3 目 and from the $\sigma$ model 11,16 agree with conventional diagrammatic perturbation theory. 1720 The equivalence of the two approaches outside the perturbative regime has been questioned 13 as a result of recent work by Zirnbauer, 10 and by Mirlin, Müller-Groeling, and Zirnbauer 11 Starting from the $\sigma$ model in the thick-wire limit, they obtained a finite limit $\langle G\rangle \rightarrow e^{2} / 2 h$ as $L / N \ell \rightarrow \infty$ in the case $\beta=4$. On the other hand, one can prove rigorously 13 that the DMPK equation gives 
$\lim _{L \rightarrow \infty}\langle G\rangle=0$ for all $\beta$. It was this puzzling contradiction which motivated us to search for a general proof of equivalence of the DMPK equation and the $\sigma$ model, without the restriction to $\beta=2$.

In this paper, we present a general proof of the equivalence of the two approaches, which applies to all three symmetry classes $\beta$, to all length scales $L$, and to the complete distribution of transmission eigenvalues described by the $p$-point functions $\rho_{p}\left(T_{1}, \ldots, T_{p}\right)=$ $(N ! /(N-p) !) \int d T_{p+1} \ldots \int d T_{N} P\left(T_{1}, \ldots, T_{N}\right)$ for arbitrary $p$. We cannot relax the assumption that the number $N$ of propagating channels in the disordered wire is $\gg 1$, since it is needed for the derivation of the one-dimensional $\sigma$ model.11 However, we can consider the $\sigma$ model formulation of a thick disordered wire which is coupled to the leads by means of a point contact with $N_{1} \leq N$ transmitted modes, 9 and show that it is mathematically equivalent to a DMPK equation for a wire with $N_{1}$ propagating channels. The equivalence proof demonstrates that $\lim _{L \rightarrow \infty}\langle G\rangle=0$ in the $\sigma$ model, in apparent contradiction with Zirnbauer's work. We have reexamined the calculation of Refs. 10 and 11, and argue that for $\beta=4$ the Kramers degeneracy of the transmission eigenvalues was not taken into account properly in the super Fourier analysis. This leads to a spurious "zero-mode", which does not decay as $L \rightarrow \infty$. Restoring Kramers degeneracy, we obtain modified expressions for $\langle G\rangle$ and var $G$ which decrease exponentially in the localized regime and moreover agree well with numerical simulations.21

Both the $\sigma$ model and the DMPK equation were derived from a number of different models for a disordered wire. The original derivation of the DMPK equation by Dorokhov, which started from a model of $N$ coupled chains with defects, was followed by the randommatrix formulation of Mello, Pereyra, and Kumar $\mathrm{\theta}$ These authors considered a product of random transfer matrices, drawn from an ensemble of maximum entropy. Later it was shown that the DMPK equation is insensitive to the choice of the ensemble, the only relevant assumptions being weak scattering (mean free path $\ell$ much greater than the Fermi wave length $\lambda_{F}$ ) and equivalence of the scattering channels.22,23 It is this latter assumption which restricts the DMPK equation to a wire geometry. From the mathematical point of view the DMPK equation is the diffusion equation on a certain coset-space of transfer matrices.24 The one-dimensional $\sigma$ model was originally derived by Efetov and Larkin6 6 G from a white noise model for the disorder potential. Two later derivations used random-matrix models for the Hamiltonian of the disordered wire. Iida, Weidenmüller, and Zuk (IWZ) adapted Wegner's $n$-orbital modeles to the study of transport properties.9 In this description, the wire is modeled by a large number of disordered segments in series, each segment having a random Hamiltonian drawn from the Gaussian ensemble. An alternative derivation of the $\sigma$ model, due to Fyodorov and Mirlin,26 uses a random band matrix to model the Hamiltonian of the disordered wire. In the present paper we follow Ref. 11 and use the IWZ formulation of the $\sigma$ model.

Our proof of equivalence of the DMPK equation and the $\sigma$ model builds on the ideas which were used by Rejaei 15 to calculate $\rho(T)$ from the $\sigma$ model for $\beta=2$. Inspired by Nazarov's diagrammatic calculation of $\rho(T)$ in the metallic regime, 27 Rejaei introduced a generating function $F$ which depends both on the transmission eigenvalues $T_{n}$ and on the radial parameters $\theta_{i}$ of the supermatrices in the unitary $\sigma$ model. Rejaei was able to solve the $1 d \sigma$ model exactly for $\beta=2$ and thus obtained the density $\rho(T)$ as a function of $L$ by taking derivatives of $F$ with respect to the $\theta_{i}$ 's. The resulting $\rho(T)$ could then be compared 
with the result from the DPMK equation.13 We introduce a more general generating function which allows us to establish the equivalence of the $\sigma$ model and the DMPK equation at the level of $p$-point functions $\rho_{p}\left(T_{1}, \ldots, T_{p}\right)$, without actually having to compute this function. This approach works also for $\beta=1$ and 4 , where no explicit solution of the $\sigma$ model is available.

The outline of the paper is as follows: In Sec. II, an outline of the equivalence proof is given. The full proof for the $\sigma$ model with $8 \times 8$ supermatrices follows in Secs. [II and IV, with technical material in Apps. A- Q. For the $p$-point functions $\rho_{p}\left(T_{1}, \ldots, T_{p}\right)$, we have to consider the $\sigma$ model with $8 p \times 8 p$ supermatrices. This extension is described in App. D. In section $\nabla$, we discuss the symplectic symmetry class $(\beta=4)$ in relation to Refs. 10 and 11. By accounting for Kramers degeneracy, we obtain modified expressions for $\langle G\rangle$ and $\operatorname{var} G$, which we compare with numerical simulations of the IWZ model by Mirlin and Müller-Groeling.21 We conclude in Sec. V1.

\section{OUTLINE OF THE EQUIVALENCE PROOF}

Although our equivalence proof is technically rather involved, the basic idea can be described in a few paragraphs. In this section, we present an outline of the equivalence proof for the small $\sigma$ model $(8 \times 8$ supermatrices). The details are given in the following two sections and in the appendices $\mathrm{A}-\mathrm{C}$. Appendix D contains the necessary modifications to extend the proof to $\sigma$ models with supermatrices of arbitrary size.

Part of the complexity of the problem is that the $\sigma$ model and the DMPK equation focus on totally different objects. In the $\sigma$ model, transport properties are expressed as functional integrals over supermatrices $Q .0 .11$ (A supermatrix is a matrix containing an equal number of commuting and anticommuting elements. We follow the notation and conventions of Refs. 8, 9, and 11.) For the small $\sigma$ model the $8 \times 8$ supermatrices are parameterized as 8.8

$$
\begin{aligned}
& Q=T^{-1} \Lambda T, \quad \Lambda=\left(\begin{array}{rr}
1 & 0 \\
0 & -1
\end{array}\right), \\
& T=\left(\begin{array}{cc}
u^{-1} & 0 \\
0 & v^{-1}
\end{array}\right) \exp \left(\begin{array}{cc}
0 & \frac{1}{2} \hat{\theta} \\
\frac{1}{2} \hat{\theta} & 0
\end{array}\right)\left(\begin{array}{ll}
u & 0 \\
0 & v
\end{array}\right),
\end{aligned}
$$

where $u$ and $v$ are pseudo-unitary $4 \times 4$ supermatrices. Notice that $Q$ satisfies the non-linear constraint $Q^{2}=1$, hence the name "non-linear" $\sigma$ model. (The letter $\sigma$ is used for historical reasons.) The $4 \times 4$ supermatrix $\hat{\theta}$ is called the radial part of $Q$. It has the form

$$
\hat{\theta}=\left(\begin{array}{cccc}
\theta_{1} & \theta_{2} & 0 & 0 \\
\theta_{2} & \theta_{1} & 0 & 0 \\
0 & 0 & i \theta_{3} & i \theta_{4} \\
0 & 0 & i \theta_{4} & i \theta_{3}
\end{array}\right)
$$

with the symmetry restrictions

$$
\begin{array}{ll}
\theta_{4}=0 & \text { if } \beta=1, \\
\theta_{2}=\theta_{4}=0 & \text { if } \beta=2, \\
\theta_{2}=0 & \text { if } \beta=4 .
\end{array}
$$


While the $\sigma$ model works with the radial part of a supermatrix, the DMPK equation works with the radial part of an ordinary matrix (containing only commuting elements). This is the transfer matrix $X$. The radial part of $X$ is an $N \times N$ diagonal matrix $\hat{\lambda}$, related to the eigenvalues of $X X^{\dagger}$. The eigenvalues of $X X^{\dagger}$ come in $N$ inverse pairs $e^{ \pm x_{n}}$, related to the diagonal elements $\lambda_{n}$ of $\hat{\lambda}$ by $\lambda_{n}=\sinh ^{2} x_{n}$. For $\beta=4$ the eigenvalues are twofold degenerate (Kramers degeneracy). The matrix $\hat{\lambda}$ then contains only the $N$ independent eigenvalues. The conductance $G$ is directly related to the $\lambda_{n}$ 's by the Landauer formula, 28

$$
G=\frac{2 e^{2}}{h} \sum_{n=1}^{N} T_{n}=\frac{2 e^{2}}{h} \sum_{n=1}^{N} \frac{1}{1+\lambda_{n}},
$$

since the $N$ independent transmission eigenvalues $T_{n}$ are related to the $\lambda_{n}$ 's by $T_{n}=(1+$ $\left.\lambda_{n}\right)^{-1}$.

We connect both approaches by considering a generating function $F(\hat{\theta}, \hat{\lambda})$ which depends on both radial matrices:

$$
\begin{aligned}
& F(\hat{\theta}, \hat{\lambda})=\prod_{n=1}^{N} f\left(\hat{\theta}, \lambda_{n}\right), \\
& f(\hat{\theta}, \lambda)=\operatorname{Sdet}^{-d / 2}\left[\lambda+\cosh ^{2}(\hat{\theta} / 2)\right]= \\
& {\left[\frac{\left(1+2 \lambda+\cos \left(\theta_{3}+\theta_{4}\right)\right)\left(1+2 \lambda+\cos \left(\theta_{3}-\theta_{4}\right)\right)}{\left(1+2 \lambda+\cosh \left(\theta_{1}+\theta_{2}\right)\right)\left(1+2 \lambda+\cosh \left(\theta_{1}-\theta_{2}\right)\right)}\right]^{d / 2},} \\
& d=1 \text { if } \beta=1,2 ; \quad d=2 \text { if } \beta=4 .
\end{aligned}
$$

The symbol Sdet stands for the superdeterminant of a supermatrix. For $\beta=2$ this is the generating function introduced by Rejaei.

An ensemble of disordered wires of length $L$ provides a distribution of $\hat{\lambda}$. The ensemble average $\langle F(\hat{\theta}, \hat{\lambda})\rangle$ contains all statistical properties that are accessible from the small $\sigma$ model. These include the average conductance $\langle G\rangle$, its variance var $G$ and the density of transmission eigenvalues $\rho(T)$. We explain in appendix A how to extract these quantities by taking derivatives of $\langle F(\hat{\theta}, \hat{\lambda})\rangle$. The average $\langle F(\hat{\theta}, \hat{\lambda})\rangle$ can be determined by each of the two approaches independently, in terms of a partial differential equation for the $L$-dependence and an initial condition at $L=0$. For the $\sigma$ model on the one hand, the evolution equation reads

$$
\frac{\partial}{\partial L}\langle F(\hat{\theta}, \hat{\lambda})\rangle=\frac{2}{\xi} \Delta_{\hat{\theta}}\langle F(\hat{\theta}, \hat{\lambda})\rangle,
$$

where $\Delta_{\hat{\theta}}$ is the (radial part of the) Laplacian on the $\sigma$ model space, and where $\xi=\beta N \ell$ is the localization length. The explicit form of $\Delta_{\hat{\theta}}$ is given by

$$
\Delta_{\hat{\theta}}=\frac{\beta}{2 d} \sum_{i} J^{-1}(\hat{\theta}) \frac{\partial}{\partial \theta_{i}} J(\hat{\theta}) \frac{\partial}{\partial \theta_{i}}
$$

where the sum runs over the independent coordinates $\theta_{i}$ [see Eq. $\left.(2.1 \mathrm{~d})\right]$ and $J(\hat{\theta})$ is the integration measure for the radial decomposition (2.1), 


$$
\begin{array}{cl}
J\left(\theta_{1}, \theta_{2}, \theta_{3}\right)=\sinh \theta_{1} \sinh \theta_{2} \sin ^{3} \theta_{3} \prod_{s_{1}, s_{2}= \pm 1} \sinh ^{-2}\left(\frac{1}{2}\left(\theta_{1}+s_{1} \theta_{2}+i s_{2} \theta_{3}\right)\right) & \text { if } \beta=1, \\
J\left(\theta_{1}, \theta_{3}\right)=\sinh \theta_{1} \sin \theta_{3} \prod_{s_{1}= \pm 1} \sinh ^{-2}\left(\frac{1}{2}\left(\theta_{1}+i s_{1} \theta_{3}\right)\right), & \text { if } \beta=2, \\
J\left(\theta_{1}, \theta_{3}, \theta_{4}\right)=\sin \theta_{3} \sin \theta_{4} \sinh ^{3} \theta_{1} \prod_{s_{1}, s_{2}= \pm 1} \sinh ^{-2}\left(\frac{1}{2}\left(\theta_{1}+i s_{1} \theta_{3}+i s_{2} \theta_{4}\right)\right) & \text { if } \beta=4 .
\end{array}
$$

The DMPK equation on the other hand, yields the evolution equation

$$
\frac{\partial}{\partial L}\langle F(\hat{\theta}, \hat{\lambda})\rangle=\frac{2}{\xi}\left\langle D_{\hat{\lambda}} F(\hat{\theta}, \hat{\lambda})\right\rangle
$$

where $D_{\hat{\lambda}}$ is a second order differential operator in the parameters $\lambda_{n}$,

$$
\begin{aligned}
D_{\hat{\lambda}} & =J^{-1}(\hat{\lambda}) \sum_{n=1}^{N} \frac{\partial}{\partial \lambda_{n}} J(\hat{\lambda}) \lambda_{n}\left(1+\lambda_{n}\right) \frac{\partial}{\partial \lambda_{n}}, \\
J(\hat{\lambda}) & =\prod_{n>m}\left|\lambda_{n}-\lambda_{m}\right|^{\beta} .
\end{aligned}
$$

The key ingredient of the equivalence proof is the identity

$$
\Delta_{\hat{\theta}} F(\hat{\theta}, \hat{\lambda})=D_{\hat{\lambda}} F(\hat{\theta}, \hat{\lambda})
$$

which shows that the evolution with $L$ of $\langle F(\hat{\theta}, \hat{\lambda})\rangle$ is the same in both approaches. Showing that the initial conditions at $L=0$ coincide as well, completes the equivalence proof.

\section{ONE-DIMENSIONAL $\sigma$ MODEL}

We begin the detailed exposition of the equivalence proof with a formulation of the $\sigma$ model. As in Ref. 11, we use the formulation of Iida-Weidenmüller-Zuk (IWZ).

\section{A. The IWZ model}

The IWZ model9 19 applies Wegner's $n$-orbital mode 25 to a wire geometry and supplements it by a coupling to ideal (not disordered) leads, as in Landauer's approach to conduction.29 The left and right leads (labeled by indices 1 and 2) contain $N_{1}$ and $N_{2}$ propagating modes each (per spin direction for $\beta=1,2$, or per Kramers doublet for $\beta=4$ ). The disordered wire of length $L$ is assumed to consist of $K$ segments in series (Fig. 1). The Hamiltonian $H$ of the disordered wire without leads is represented by a matrix $H_{\mu \nu}^{i j}$, where the upper indices $i, j$ label the segments $1 \leq i, j \leq K$ and the lower indices $\mu, \nu$ label the $M$ states (per spin direction or Kramers doublet) within each segment. The elements of $H$ are real $(\beta=1)$, complex $(\beta=2)$ or quaternion $(\beta=4)$ numbers. The coupling between the states inside one segment is described by the matrices $H_{\mu \nu}^{i i}$, which are distributed according to the Gaussian ensemble

$$
P\left(H^{i i}\right)=\text { const. } \times \exp \left(-\frac{1}{4} \beta M v_{1}^{-2} \operatorname{Tr}\left(H^{i i}\right)^{2}\right) .
$$


Here $v_{1}$ is a parameter which governs the level density at the Fermi level $(E=0)$. The coupling between the states of adjacent segments is given by another set of Gaussian distributed random matrices $H^{i j}=\left(H^{j i}\right)^{\dagger}$ (with coupling parameter $v_{2}$ ),

$$
\begin{aligned}
& P\left(H^{i j}\right)=\text { const. } \times \exp \left(-\frac{1}{2} \beta M^{2} v_{2}^{-2} \operatorname{Tr} H^{i j} H^{j i}\right), \\
& j=i \pm 1 .
\end{aligned}
$$

Segments which are not adjacent are uncoupled, $H^{i j}=0$ if $|i-j| \geq 2$. The coupling to the ideal leads is described by a fixed $K M \times\left(N_{1}+N_{2}\right)$ rectangular matrix $W=W_{1}+W_{2}$ with real $(\beta=1)$, complex $(\beta=2)$ or quaternion $(\beta=4)$ elements. The matrix $W$ has elements $W_{\mu n}^{i}$, where $i$ labels the segment, $\mu$ the states in the segment, and $n$ the modes in the leads. The elements of $W_{1}$ (which describes the coupling to lead 1) are nonzero only for $i=1$ and $1 \leq n \leq N_{1}$; the elements of $W_{2}$ (coupling to lead 2) are nonzero only for $i=K$ and $N_{1}<n \leq N_{1}+N_{2}$.

The scattering matrix $S$ (matrix elements $S_{n m}$ ) of the system at energy $E$ is given by

$$
S=1-2 \pi i W^{\dagger}\left(E-H+i \pi W W^{\dagger}\right)^{-1} W
$$

The indices $n, m$ correspond to lead 1 if $1 \leq n, m \leq N_{1}$ and to lead 2 if $N_{1}<n, m \leq N_{1}+N_{2}$. The reflection and transmission matrices $r, r^{\prime}, t, t^{\prime}$ are submatrices of $S$,

$$
S=\left(\begin{array}{ll}
r & t^{\prime} \\
t & r^{\prime}
\end{array}\right) \text {. }
$$

Since $S$ is unitary, the products $t^{\dagger} t$ and $t^{\prime \dagger} t^{\prime}$ have the same set of non-zero eigenvalues, denoted by $T_{n}=\left(1+\lambda_{n}\right)^{-1}$. (If $N_{2}>N_{1}$ there are also $N_{2}-N_{1}$ transmission eigenvalues which are zero, and can therefore be disregarded.)

\section{B. The generating function}

We now define the generating function $F(\hat{\theta}, \hat{\lambda})$ introduced in the previous section. We start from the the relationship (3.3) between the scattering matrix and the Hamiltonian in the IWZ model. We consider the generating function

$$
\begin{aligned}
& F=\operatorname{Sdet}^{-\frac{1}{2}}\left(E-\mathcal{H}+i \pi W_{1} W_{1}^{\dagger} Q+i \pi W_{2} W_{2}^{\dagger} \Lambda\right), \\
& \mathcal{H}=H 1_{8} \quad \text { if } \beta=1,4 ; \quad \mathcal{H}=(\operatorname{Re} H) 1_{8}+i(\operatorname{Im} H) \tau_{3} \quad \text { if } \beta=2 .
\end{aligned}
$$

Here $1_{8}$ is the $8 \times 8$ supersymmetric unit matrix and $\tau_{3}$ is a diagonal matrix with elements $(1,-1,1,-1,1,-1,1,-1)$. The matrix $\Lambda$ was defined in Eq. 2.1a). Note that $Q$ is an arbitrary supermatrix as in Eq. (2.1) and that it replaces the matrix $\Lambda$ in the coupling term of lead 1. In App. $B$ we show that $F$ depends only on the radial part $\hat{\theta}$ of the matrix $Q$ and that the only dependence on $H$ is through the transmission eigenvalues $T_{n}=\left(1+\lambda_{n}\right)^{-1}$. We also show that Eq. (3.5) reduces to the function $F(\hat{\theta}, \hat{\lambda})$ defined in Eq. (2.3) of the previous section. 
In the following, we evaluate the ensemble average $\langle F\rangle$ using the supersymmetric formalism. We first express $\langle F\rangle$ as a Gaussian integral over an $8 M K$-dimensional supervector $\psi$ :

$$
\langle F\rangle=\left\langle\int \mathcal{D} \psi \exp \left(\frac{1}{2} i \psi^{\dagger} \Lambda\left(E-\mathcal{H}+i \pi W_{1} W_{1}^{\dagger} Q+i \pi W_{2} W_{2}^{\dagger} \Lambda+i \epsilon \Lambda\right) \psi\right)\right\rangle .
$$

The convergence of the Gaussian integral is assured by the parameterization (2.1) of the matrix $Q$. Performing the standard steps, described in Refs. 9 and 11, we obtain in the relevant limit $M \rightarrow \infty$

$$
\begin{aligned}
\langle F\rangle & =\int d Q_{1} \int d Q_{K} f_{1}\left(Q, Q_{1}\right) f_{2}\left(\Lambda, Q_{K}\right) W\left(Q_{1}, Q_{K}\right), \\
W\left(Q_{1}, Q_{K}\right) & =\int d Q_{2} \ldots \int d Q_{K-1} \exp \left(-\frac{d v_{2}^{2}}{2 v_{1}^{2}} \sum_{i=1}^{K-1} \operatorname{Str}\left(Q_{i} Q_{i+1}\right),\right) \\
f_{1}\left(Q, Q_{1}\right) & =\exp \left(-\frac{1}{2} d \sum_{n=1}^{N_{1}} \operatorname{Str} \ln \left(1+x_{n} Q Q_{1}\right)\right), \\
f_{2}\left(Q, Q_{K}\right) & =\exp \left(-\frac{1}{2} d \sum_{n=N_{1}+1}^{N_{1}+N_{2}} \operatorname{Str} \ln \left(1+x_{n} Q Q_{K}\right)\right) .
\end{aligned}
$$

The numbers $x_{n}$ denote the eigenvalues of the matrices $\left(\pi / v_{1}\right) W_{1}^{\dagger} W_{1}$ (if $1 \leq n \leq N_{1}$ ) or $\left(\pi / v_{1}\right) W_{2}^{\dagger} W_{2}$ (if $\left.N_{1}<n \leq N_{1}+N_{2}\right)$. The integer $d$ was defined in Eq. (2.3d).

Following Ref. 11, we consider the limit $v_{1}^{2} \ll v_{2}^{2}$. Then the sum in (3.7b) can be replaced by an integral and the $Q_{i}$-integrals yield a path integral. The discrete number of segments $K$ becomes the continuous (dimensionless) variable $s$. The propagator (3.7b) can be identified with the heat kernel of the supersymmetric space, determined by the heat equation 11

$$
\begin{aligned}
2 \beta\left(v_{2} / v_{1}\right)^{2} \frac{\partial}{\partial s} W\left(Q^{\prime}, Q^{\prime \prime}\right) & =\Delta_{Q^{\prime}} W\left(Q^{\prime}, Q^{\prime \prime}\right), \\
\lim _{s \rightarrow 0} W\left(Q^{\prime}, Q^{\prime \prime}\right) & =\delta\left(Q^{\prime}, Q^{\prime \prime}\right) .
\end{aligned}
$$

The precise definition of the Laplacian $\Delta_{Q}$ and the detailed justification of Eq. (3.8) are contained in Ref. 11 ( $\Delta_{Q}$ in Eq. (3.8) differs by an additional factor $\beta /(8 d)$ with respect to the notations of Ref. 11). We thus arrive at the expression

$$
\langle F\rangle=\int d Q^{\prime} \int d Q^{\prime \prime} f_{1}\left(Q, Q^{\prime}\right) W\left(Q^{\prime}, Q^{\prime \prime}\right) f_{2}\left(\Lambda, Q^{\prime \prime}\right) .
$$

The next step is to notice that $f_{1}\left(Q, Q^{\prime}\right)$ has the same symmetry as the heat kernel, i.e. $f_{1}\left(T^{-1} Q T, T^{-1} Q^{\prime} T\right)=f_{1}\left(Q, Q^{\prime}\right)$ where $T$ is an arbitrary element as described in (2.1a). This implies $\Delta_{Q^{\prime}} f_{1}\left(Q, Q^{\prime}\right)=\Delta_{Q} f_{1}\left(Q, Q^{\prime}\right)$ and hence $\langle F\rangle$ also satisfies the heat equation

$$
2 \beta\left(v_{2} / v_{1}\right)^{2} \frac{\partial}{\partial s}\langle F\rangle=\Delta_{Q}\langle F\rangle .
$$

Since $\langle F\rangle$ only depends on the radial part $\hat{\theta}$ of $Q$, it is sufficient to consider the radial part $\Delta_{\hat{\theta}}$ of the Laplacian $\Delta_{Q}$. This radial part $\Delta_{\hat{\theta}}$ can be written as in Eq. (2.4b). We thus find 
that the ensemble average $\langle F(\hat{\theta}, \hat{\lambda})\rangle$ of the generating function defined in Eq. (2.3) satisfies the partial differential equation

$$
2 \beta\left(v_{2} / v_{1}\right)^{2} \frac{\partial}{\partial s}\langle F(\hat{\theta}, \hat{\lambda})\rangle=\Delta_{\hat{\theta}}\langle F(\hat{\theta}, \hat{\lambda})\rangle,
$$

with the initial condition implied by Eq. (3.8),

$$
\lim _{s \rightarrow 0}\langle F(\hat{\theta}, \hat{\lambda})\rangle=\int d Q^{\prime} f_{1}\left(Q, Q^{\prime}\right) f_{2}\left(\Lambda, Q^{\prime}\right) .
$$

Together, Eqs. (3.11) and (3.12) determine the ensemble average of the generating function $F(\hat{\theta}, \hat{\lambda})$ evaluated in the framework of the nonlinear $\sigma$ model.

The two limits of the IWZ model which were needed for the derivation of Eq. (3.12), $M \rightarrow \infty$ and $v_{1}^{2} / v_{2}^{2} \rightarrow 0$, restrict the validity of Eq. (3.12) to the case of weak disorder $\left(\ell \gg \lambda_{F}\right)$ and thick wires $(N \gg 1)$ respectively. 111 Whereas the requirement of weak disorder is also needed for the DMPK equation, the requirement that the number of channels in the disordered wire be large is not. To see how the latter requirement follows from the condition $v_{1}^{2} \ll v_{2}^{2}$, we consider the expression for the average conductance $\langle G\rangle$ in the diffusive metallic regime $(\ell \ll L \ll N \ell)$, 11

$$
\langle G\rangle=\frac{2 e^{2}}{h} \frac{N \ell}{L}=\frac{2 e^{2}}{h} \frac{4 v_{2}^{2}}{v_{1}^{2} s} .
$$

Taking the linear dimension of a segment of the disordered wire in the IWZ model of order $\ell$ (i.e. $s \approx L / \ell$, see Ref. 9), we find that $v_{1}^{2} \ll v_{2}^{2}$ corresponds to $N \gg 1$. However, no restriction has been put to the numbers $N_{1}$ and $N_{2}$ of propagating channels in the leads in the above derivation of the $\sigma$ model, which allows us to consider finite values of $N_{1}$ and $N_{2}$. This situation corresponds to the case in which the thick disordered wire is coupled to the leads 1 and 2 by means of point contacts, with $N_{1}, N_{2}$ open channels. As in Ref. 11, the case of a disordered wire without point contacts is recovered in the limit $N_{1}, N_{2} \rightarrow \infty$.

We conclude this section with some remarks about the choice of initial conditions. In usual $\sigma$ model calculations, 19, 11,15 one considers ideal coupling $\left(x_{n}=1, n=1, \ldots, N_{1}+N_{2}\right)$ and identifies $N=N_{1}=N_{2}$ (equal number of channels in the leads and in the wire). In the thick-wire limit $N \rightarrow \infty$ the function $f_{i}\left(Q, Q^{\prime}\right)$ is just the delta function $11 \delta\left(Q, Q^{\prime}\right)$, and $\langle F\rangle$ becomes identical to the heat kernel itself [cf. Eq. (3.9)]:

$$
\langle F\rangle=W(Q, \Lambda), N_{1}=N_{2}=N \gg 1 \text {. }
$$

For $\beta=2$, this result was derived by Rejaei. 1 In this case $\langle F\rangle$ has the delta-function initial condition $\lim _{s \rightarrow 0}\langle F\rangle=\delta(Q, \Lambda)$. To make contact with the DMPK equation, we need a different "ballistic" initial condition, such that all $T_{n}$ 's are unity in the limit of zero wire length. To achieve this, we take ideal coupling and assume that one of the leads has many more channels than the other. To be specific, we fix $N_{1}$ and take the limit $N_{2} \rightarrow \infty$. One then finds the initial condition

$$
\begin{aligned}
& \lim _{s \rightarrow 0}\langle F\rangle=\exp \left(-\frac{1}{2} N_{1} d \operatorname{Str} \ln (1+Q \Lambda)\right) \\
& =\left(\frac{\cos \theta_{3}+\cos \theta_{4}}{\cosh \theta_{1}+\cosh \theta_{2}}\right)^{N_{1} d}, 1 \leq N_{1} \ll N_{2} .
\end{aligned}
$$


In the next section, we will see that this is precisely the ballistic initial condition of the DMPK equation.

\section{DMPK EQUATION}

Let us now evaluate the ensemble average of the generating function (2.3) from the DMPK equation. The DMPK equation is a Fokker-Planck-type equation for the $L$-evolution of the probability distribution $P(\hat{\lambda})$ of the $\lambda_{n}$ 's: 15

$$
\begin{aligned}
& \frac{1}{2}(\beta N+2-\beta) \ell \frac{\partial}{\partial L} P(\hat{\lambda})= \\
& \sum_{n=1}^{N} \frac{\partial}{\partial \lambda_{n}} \lambda_{n}\left(1+\lambda_{n}\right) J(\hat{\lambda}) \frac{\partial}{\partial \lambda_{n}} J^{-1}(\hat{\lambda}) P(\hat{\lambda}), \\
& J(\hat{\lambda})=\prod_{n>m}\left|\lambda_{n}-\lambda_{m}\right|^{\beta},
\end{aligned}
$$

where $\ell$ denotes the mean free path in the disordered wire and $N$ the number of propagating modes. There is no restriction to $N \gg 1$ in the DMPK approach. We take the ballistic initial condition

$$
\lim _{L \rightarrow 0} P(\hat{\lambda})=\prod_{n=1}^{N} \delta\left(\lambda_{n}-0^{+}\right) .
$$

The DMPK equation implies for $F(\hat{\theta}, \hat{\lambda})$ the evolution equation国

$$
\begin{aligned}
\frac{\partial}{\partial L}\langle F(\hat{\theta}, \hat{\lambda})\rangle & =\frac{\partial}{\partial L} \int d \lambda_{1} \ldots \int d \lambda_{N} F(\hat{\theta}, \hat{\lambda}) P(\hat{\lambda}) \\
& =\frac{2}{\ell}(\beta N+2-\beta)^{-1}\left\langle D_{\hat{\lambda}} F(\hat{\theta}, \hat{\lambda})\right\rangle,
\end{aligned}
$$

with the differential operator $D_{\hat{\lambda}}$ given by Eq. (2.5b). In Appendix $\mathrm{Q}$ we prove the algebraic identity between the two different types of Laplacians (2.4b) and (2.5b) applied to $F(\hat{\theta}, \hat{\lambda})$,

$$
\Delta_{\hat{\theta}} F(\hat{\theta}, \hat{\lambda})=D_{\hat{\lambda}} F(\hat{\theta}, \hat{\lambda})
$$

From Eqs. (4.3), and (4.4) we conclude that that the average $\langle F(\hat{\theta}, \hat{\lambda})\rangle$, calculated in the framework of the DMPK equation, also fulfills the evolution equation (3.11) of the nonlinear $\sigma$ model, provided we identify [cf. Eq. (3.13)]

$$
\frac{4}{s}\left(v_{2} / v_{1}\right)^{2}=\frac{N \ell}{L}=\frac{\xi}{\beta L}, \quad N \gg 1
$$

Here we introduced the localization length $\xi=\beta N \ell$ (notice that the definition of $\xi$ in Ref. 11 differs by a factor $2 / \beta$ ).

It remains to compare the initial conditions. The ballistic initial condition for the DMPK equation implies 


$$
\lim _{L \rightarrow 0}\langle F(\hat{\theta}, \hat{\lambda})\rangle=f(\hat{\theta}, \lambda=0)^{N}=\left(\frac{\cos \theta_{3}+\cos \theta_{4}}{\cosh \theta_{1}+\cosh \theta_{2}}\right)^{N d},
$$

which equals the initial condition (3.15) for the nonlinear $\sigma$ model (The thick-wire limit $\lim _{L \rightarrow 0}\langle F\rangle=\delta(Q, \Lambda)$ is obtained by letting $N \rightarrow \infty$ in the above expression). This proves the equivalence of both approaches, as far as the generating function (2.3) is concerned. In Appendix $\mathrm{D}$ we extend the equivalence proof to $p$-point functions $\rho_{p}\left(T_{1}, \ldots, T_{p}\right)$ for arbitrary $p$.

\section{THE CONTROVERSIAL SYMPLECTIC ENSEMBLE}

The main motivation of this work was to resolve a controversy between the DMPK equation and the one-dimensional $\sigma$ model in the symplectic symmetry class $(\beta=4)$. On the one hand, the DMPK equation implies $13\langle G\rangle \rightarrow 0$ as $L \rightarrow \infty$. On the other hand, Zirnbauer 10 finds from the $\sigma$ model that $\langle G\rangle \rightarrow \frac{1}{2} e^{2} / h$ as $L \rightarrow \infty$.

The equivalence proof presented in this paper has as a logical consequence that $\langle G\rangle \rightarrow 0$ as $L \rightarrow \infty$ if $\langle G\rangle$ is evaluated in the framework of the $\sigma$ model. To demonstrate this, we apply the argument of Ref. 13. The DMPK equation implies for the average dimensionless conductance $g=\sum_{n}\left(1+\lambda_{n}\right)^{-1}$ the evolution equation $\theta$

$$
\xi \frac{\partial\langle g\rangle}{\partial L}=-\beta\left\langle g^{2}\right\rangle-(2-\beta)\left\langle g_{2}\right\rangle
$$

with $g_{2}=\sum_{n}\left(1+\lambda_{n}\right)^{-2}$. This relation also follows from the evolution equation (3.11) of the $\sigma$ model (expanding the generating function for small $\theta_{i}$ and applying the results of appendix A). Since $0 \leq g_{2} \leq g^{2}$, we have

$$
\xi \frac{\partial\langle g\rangle}{\partial L} \leq-\frac{1}{2} \beta\left\langle g^{2}\right\rangle \leq 0
$$

We suppose that $\lim _{L \rightarrow \infty}\langle g\rangle$ exists. Since $\partial\langle g\rangle / \partial L \leq 0$ [Eq. (5.2)] this implies $\lim _{L \rightarrow \infty} \partial\langle g\rangle / \partial L=0$. Hence $\lim _{L \rightarrow \infty}\left\langle g^{2}\right\rangle=0$ by Eq. (5.2). Since $\langle g\rangle^{2} \leq\left\langle g^{2}\right\rangle$ this implies that also $\lim _{L \rightarrow \infty}\langle g\rangle=0$.

Where does the non-zero limit in Refs. 10 and 11 come from? The ground-breaking contribution of Zirnbauer was to use a "super-Fourier expansion" of the heat kernel $W\left(Q, Q^{\prime}\right)$ in terms of eigenfunctions of the Laplacian in the space of the $\sigma$ model. This type of Fourier analysis is well understood for classical symmetric spaces. 30 The development and application of the supersymmetric analogue for the $\sigma$ model enabled Zirnbauer, Mirlin, and Müller-Groeling to compute non-perturbatively the first two moments of the conductance for any $\beta$. The non-zero limiting value $\lim _{L \rightarrow \infty}\langle g\rangle=1 / 4$ for $\beta=4$ resulted from a "zero mode", a non-trivial eigenfunction of the Laplacian with zero eigenvalue. Since this zero mode does not decay as $L \rightarrow \infty$, it led to the surprising conclusion of absence of localization in a wire with spin-orbit scattering in zero magnetic field 10

An explicit expression for the zero-mode was not obtained in Refs. 10 and 11, but only its contribution to the moments of the conductance was computed. By inspecting the initial condition (3.15) of the generating function for the $\sigma$ model we have been able to construct 
a zero mode for $\beta=4$, but only if we ignore the Kramers degeneracy of the transmission eigenvalues. This unphysical zero mode, given by

$$
\phi_{0}\left(\theta_{1}, \theta_{3}, \theta_{4}\right)=\frac{\cos \theta_{3}+\cos \theta_{4}}{2+2 \cosh \theta_{1}}
$$

arises by taking the initial condition (3.15) with $N_{1}=1$ and $\beta=4$, but without the extra factor two in the exponent, required by Kramers degeneracy. This unphysical initial condition solves the evolution equation (3.11) for the ensemble average of the generating function and implies an $L$-independent average conductance $\langle g\rangle=1 / 4$. Although we can not prove that Eq. (5.3) is Zirnbauer's zero mode, the coincidence with the limiting value $\lim _{L \rightarrow \infty}\langle g\rangle=1 / 4, \lim _{L \rightarrow \infty} \operatorname{var} g=1 / 16$ is quite suggestive.

The reason why we have to exclude the zero mode (5.3) from the Fourier expansion of the heat kernel is that it is not single-valued on the $\sigma$ model space of supermatrices $Q$, although it is a well-defined function of $\hat{\theta}$. The parameterization (2.1) of $Q$ is $2 \pi$-periodic in the angles $\theta_{ \pm}=\theta_{3} \pm \theta_{4}$. We can then consider on the space of angles $\theta_{3}, \theta_{4}$ a parity operation $P$ which consists of adding $\pi$ to both of these angles. This parity operation does not change $Q$, but it changes the zero mode (5.3). The Laplacian (2.4b) commutes with $P$ and the eigenfunctions have therefore either even or odd parity (eigenvalues +1 or -1 of $P$, respectively). The physical modes of the $\sigma$ model must have even parity, since only these functions are single-valued. For $\beta=4$, it is the Kramers degeneracy which ensures that the initial condition (3.15) has even parity.

This observation led us to check the parity of the eigenfunctions $\phi_{\nu}(Q)$ of the Laplacian in the super Fourier analysis of Refs. 10 and 11. We consider the eigenvalue equation

$$
\Delta_{\hat{\theta}} \phi_{\nu}\left(\theta_{1}, \theta_{3}, \theta_{4}\right)=-\varepsilon(\nu) \phi_{\nu}\left(\theta_{1}, \theta_{3}, \theta_{4}\right)
$$

for $\beta=4$ in the limit $\theta_{1} \rightarrow \infty$ at fixed $\theta_{3}, \theta_{4}$. In this limit, the Laplace operator simplifies considerably

$$
\Delta_{\hat{\theta}} \rightarrow e^{\theta_{1}} \frac{\partial}{\partial \theta_{1}} e^{-\theta_{1}} \frac{\partial}{\partial \theta_{1}}+\frac{1}{\sin \theta_{3}} \frac{\partial}{\partial \theta_{3}} \sin \theta_{3} \frac{\partial}{\partial \theta_{3}}+\frac{1}{\sin \theta_{4}} \frac{\partial}{\partial \theta_{4}} \sin \theta_{4} \frac{\partial}{\partial \theta_{4}} .
$$

From this expression one may identify the set of quantum numbers $\nu=\left(\lambda, 1+2 n_{1}, 1+2 n_{2}\right)$, where $\lambda$ is a real number and $n_{1}, n_{2}$ are non-negative integers. The asymptotic behavior of the eigenfunctions $\phi_{\nu}\left(\theta_{1}, \theta_{3}, \theta_{4}\right)$ is given by

$$
\phi_{\nu}\left(\theta_{1}, \theta_{3}, \theta_{4}\right) \sim \exp \left[\frac{1}{2}(1+i \lambda) \theta_{1}\right]\left(P_{n_{1}}\left(\cos \theta_{3}\right) P_{n_{2}}\left(\cos \theta_{4}\right)+P_{n_{2}}\left(\cos \theta_{3}\right) P_{n_{1}}\left(\cos \theta_{4}\right)\right),
$$

with the Legendre polynomials $P_{n}(x)$ and the eigenvalues

$$
\varepsilon\left(\lambda, 1+2 n_{1}, 1+2 n_{2}\right)=\frac{1}{4}\left(\lambda^{2}+\left(1+2 n_{1}\right)^{2}+\left(1+2 n_{2}\right)^{2}-1\right) .
$$

The parity of this eigenfunction is just $(-1)^{n_{1}+n_{2}}$ and we have to restrict ourselves to those $n_{1}$ and $n_{2}$ with $n_{1}+n_{2}$ even. Applying this selection rule to the expressions for $\langle g\rangle$ and $\left\langle g^{2}\right\rangle$ of Refs. 10 and 11, omitting the zero mode [and the subsidiary series with quantum numbers 
$\nu=(i, l, l \pm 2)$ of Refs. 10 and 11, for which the asymptotic behavior (5.6) is also valid], and multiplying the surviving terms with a factor of 2 to account for Kramers degeneracy, yields for $\beta=4$ and in the limit $N_{1}=N_{2}=N \rightarrow \infty$ the expression

$$
\begin{aligned}
\left\langle g^{n}\right\rangle= & 2^{5-n} \sum_{\substack{l_{1}, l_{2}=1,3,5, \ldots, l_{1}+l_{2} \equiv 2(\bmod 4)}} \int_{0}^{\infty} d \lambda \lambda\left(\lambda^{2}+1\right) \tanh (\pi \lambda / 2) l_{1} l_{2} p_{n}\left(\lambda, l_{1}, l_{2}\right) \\
& \times \prod_{\sigma, \sigma_{1}, \sigma_{2}= \pm 1}\left(-1+i \sigma \lambda+\sigma_{1} l_{1}+\sigma_{2} l_{2}\right)^{-1} \exp \left[-\left(\lambda^{2}+l_{1}^{2}+l_{2}^{2}-1\right) L /(2 \xi)\right],
\end{aligned}
$$

where $n=1,2$ and

$$
\begin{aligned}
& p_{1}\left(\lambda, l_{1}, l_{2}\right)=\lambda^{2}+l_{1}^{2}+l_{2}^{2}-1 \\
& p_{2}\left(\lambda, l_{1}, l_{2}\right)=\frac{1}{4}\left[2 \lambda^{4}+l_{1}^{4}+l_{2}^{4}+3 \lambda^{2}\left(l_{1}^{2}+l_{2}^{2}\right)-2 \lambda^{2}+l_{1}^{2}+l_{2}^{2}-2\right] .
\end{aligned}
$$

Note that in our notations the dimensionless conductance $g$ is by a factor 2 smaller than $g$ in the notations of Ref. 11. Comparison of Eq. (5.8) with the $\beta=4$ result of Ref. 11, where the parity selection rule was not implemented, shows that the perturbation expansion around $L / \xi=0$ is the same. (We checked this numerically up to order $(L / \xi)^{3}$.) Outside the perturbative regime, the two expressions are completely different. Instead of a non-zero limit $\langle g\rangle=1 / 4$ for $L / \xi \gg 1$, we find from Eq. (5.8) the exponential decay

$$
\langle g\rangle \approx \frac{16}{9}(2 L / \pi \xi)^{-3 / 2} e^{-L / 2 \xi} .
$$

To test our result, we have compared it with a direct numerical simulation of the IWZ model by Mirlin and Müller-Groeling21 (with $M=100, N=25$ and an average over 100 different samples). The comparison is shown in Figs. 2 and 3. It is clear that our Eq. (5.8) (solid curve) agrees quite well with the simulation, while the result of Ref. 11 does not (dotted curve).

Notice that this issue of the parity of the eigenfunctions does not occur for $\beta=1,2$, since there is only one compact angle $\left(\theta_{3}\right)$ in those cases. The parity operation on the $\hat{\theta}$-matrices exists only for $\beta=4$. For completeness we collect in Figs. 14 and 5 the results for $\langle g\rangle$ and var $g$ for all three symmetry classes. The $\beta=1,2$ results are from Ref. 11, the $\beta=4$ result is our Eq. (5.8).

\section{CONCLUSION}

We have established the exact mathematical equivalence of the two non-perturbative theoretical approaches to phase-coherent transport and localization in disordered wires: The Fokker-Planck equation of Dorokhov, Mello Pereyra, and Kumartit and the onedimensional supersymmetric nonlinear $\sigma$ model.6.6. consequence that the absence of localization in the symplectic symmetry class, obtained by Zirnbauer by super-Fourier analysis of the $\sigma$ model, is not correct. By applying a selection rule enforced by Kramers degeneracy to the eigenfunctions of Refs. 10 and 11, we have obtained modified expressions for $\langle G\rangle$ and $\operatorname{var} G$, which decay exponentially as $L \rightarrow \infty$ and

which agree well with existing numerical simulations.21 
Our equivalence proof has both conceptual and practical implications. The DMPK equation and the $1 d \sigma$ model originated almost simultaneously in the early eighties, and at the same institute.1. the next decade. Knowing that, instead of two theories, there is only one, seems to us a considerable conceptual simplification of the field. It implies that the microscopic derivations and random-matrix models developed for the $\sigma$ model apply as well to the DMPK equation, and vice versa. (we see only the restriction, that the $\sigma$ model requires the thick-wire limit $N \rightarrow \infty$, while the DMPK equation applies to any number of channels $N$.) Practically, each of the two approaches has its own advantages, and now that we know that they are equivalent, we can choose the approach which is best suited to our needs and skills.

\section{ACKNOWLEDGMENTS}

We thank C. W. J. Beenakker, B. Rejaei, and M. R. Zirnbauer for fruitful discussions. We are very grateful to A. D. Mirlin and A. Müller-Groeling for providing us with unpublished data from their numerical simulation of the IWZ model. This work was supported by the "Stichting voor Fundamenteel Onderzoek der Materie" (FOM) and by the "Nederlandse organisatie voor Wetenschappelijk Onderzoek" (NWO) (P. W. B.) and by the Human Capital Mobility program of the European Community "Quantum Dynamics of Phase-Coherent Structures" (K. F.).

\section{APPENDIX A: TRANSPORT PROPERTIES DETERMINED BY THE GENERATING FUNCTION}

We list the transport properties of interest that can be generated from $F(\hat{\theta}, \hat{\lambda})$, following Rejaei.15 Let us consider the function

$$
f\left(z_{1}, z_{2}\right)=\left\langle\frac{\operatorname{det}\left(1+z_{2} t^{\dagger} t\right)}{\operatorname{det}\left(1+z_{1} t^{\dagger} t\right)}\right\rangle,
$$

which equals $\langle F(\hat{\theta}, \hat{\lambda})\rangle$ at $z_{2}=-\sin ^{2}\left(\frac{1}{2} \theta_{3}\right), z_{1}=\sinh ^{2}\left(\frac{1}{2} \theta_{1}\right)$, and $\theta_{2}=\theta_{4}=0$. We write Eq. (A1) in the form

$$
f\left(z_{1}, z_{2}\right)=\left\langle\exp \left[\operatorname{Tr} \ln \left(1+z_{2} t^{\dagger} t\right)-\operatorname{Tr} \ln \left(1+z_{1} t^{\dagger} t\right)\right]\right\rangle .
$$

The standard expansion 89 with respect to small $z_{1}$ and $z_{2}$ yields the first two moments of the dimensionless conductance $g=(1 / d) \operatorname{Tr} t^{\dagger} t$ (with $d=1+\delta_{4, \beta}$ ),

$$
\begin{aligned}
\langle g\rangle & =\left.\frac{1}{d} \frac{\partial}{\partial z_{2}} f\left(z_{1}, z_{2}\right)\right|_{z_{1}=0=z_{2}} \\
& =-\left.\frac{1}{d} \frac{\partial}{\partial z_{1}} f\left(z_{1}, z_{2}\right)\right|_{z_{1}=0=z_{2}}, \\
\left\langle g^{2}\right\rangle & =-\left.\frac{1}{d^{2}} \frac{\partial}{\partial z_{1}} \frac{\partial}{\partial z_{2}} f\left(z_{1}, z_{2}\right)\right|_{z_{1}=0=z_{2}}
\end{aligned}
$$


We may also consider 1527 derivatives of $f\left(z_{1}, z_{2}\right)$ at $z_{1}=z_{2}$. This may require the analytic continuation of $\theta_{1}, \theta_{3}$ to complex values if $z_{1}<0, z_{2}>0$ or $z_{2}<-1$. Therefore, we introduce the function $f\left(z_{1}\right)$ as

$$
\begin{aligned}
f\left(z_{1}\right) & =\left.\frac{\partial}{\partial z_{2}} f\left(z_{1}, z_{2}\right)\right|_{z_{2}=z_{1}} \\
& =\left\langle\operatorname{Tr}\left[\left(1+z_{1} t^{\dagger} t\right)^{-1} t^{\dagger} t\right]\right\rangle \\
& =\sum_{n=0}^{\infty}\left(-z_{1}\right)^{n}\left\langle\operatorname{Tr}\left(t^{\dagger} t\right)^{n+1}\right\rangle \\
& =z_{1}^{-1}\left(\operatorname{Tr}(1)-\left\langle\operatorname{Tr}\left[\left(1+z_{1} t^{\dagger} t\right)^{-1}\right]\right\rangle\right) .
\end{aligned}
$$

The average density of transmission eigenvalues now follows from:

$$
\begin{aligned}
\rho(T) & =\left\langle\operatorname{Tr} \delta\left(T-t^{\dagger} t\right)\right\rangle \\
& =-\frac{1}{\pi T^{2}} \operatorname{Im} f\left(-\left(T+i 0^{+}\right)^{-1}\right) .
\end{aligned}
$$

The application of Eq. (A6) requires the analytical continuation of both variables $z_{1}$ and $z_{2}$ to values $<-1$.

\section{APPENDIX B: THE GENERATING FUNCTION IN TERMS OF THE TRANSMISSION MATRIX}

In this appendix, we show that Eq. (3.5) for the generating function in the IWZ model equals Eq. (2.3). We first consider the two cases $\beta=1,4$ of time reversal symmetry, when $\mathcal{H}=H 1_{8}$ in Eq. (3.5a). The necessary modifications for $\beta=2$ are described at the end.

We make use of the folding identity

$$
\operatorname{Sdet}\left(\begin{array}{cc}
1_{n} & A \\
B & 1_{m}
\end{array}\right)=\operatorname{Sdet}\left(1_{n}-A B\right)=\operatorname{Sdet}\left(1_{m}-B A\right) .
$$

We abbreviate $G_{ \pm}=\left(E-H \pm i \pi W W^{\dagger}\right)^{-1}$. Taking out the factor $\left(E-\mathcal{H}+i \pi W W^{\dagger} \Lambda\right)$ (with unit superdeterminant), we may rewrite Eq. (3.5a) as

$$
\begin{aligned}
F & =\operatorname{Sdet}^{-\frac{1}{2}}\left(1+\left(\begin{array}{cc}
G_{+} & 0 \\
0 & G_{-}
\end{array}\right) i \pi W_{1} W_{1}^{\dagger}(Q-\Lambda)\right) \\
& =\operatorname{Sdet}^{-\frac{1}{2}}\left(1+i \pi\left(\begin{array}{cc}
W_{1}^{\dagger} G_{+} W_{1} & 0 \\
0 & W_{1}^{\dagger} G_{-} W_{1}
\end{array}\right)(Q-\Lambda)\right),
\end{aligned}
$$

where we have applied Eq. (B1) on $B=W_{1}^{\dagger}$. We now insert the reflection matrix $r=$ $1-2 \pi i W_{1}^{\dagger} G_{+} W_{1}$ [see Eqs. (3.4) and (3.3)] into Eq. (B2) and obtain

$$
F=\operatorname{Sdet}^{-\frac{1}{2}}\left(\frac{1}{2}(1+\Lambda Q)+\frac{1}{2}\left(\begin{array}{cc}
r & 0 \\
0 & r^{\dagger}
\end{array}\right)(1-\Lambda Q)\right) .
$$


Now we use the parameterization (2.1) for $Q$. Notice that Eq. (B33) does not depend on the angular part of $Q$ [the matrices $u, v$ in (2.1b)]. Hence we may choose $Q$ as

$$
Q=T^{-1} \Lambda T, T=\exp \left(\frac{1}{2} \Theta\right), \Theta=\left(\begin{array}{cc}
0 & \hat{\theta} \\
\hat{\theta} & 0
\end{array}\right),
$$

which leads to

$$
\frac{1}{2}(1+\Lambda Q)=\cosh \left(\frac{1}{2} \Theta\right) T, \quad \frac{1}{2}(1-\Lambda Q)=-\sinh \left(\frac{1}{2} \Theta\right) T .
$$

Inserting this in Eq. (B3) and taking out the factor $T$ (with unit superdeterminant), we get

$$
\begin{aligned}
F & =\operatorname{Sdet}^{-1 / 2}\left(\begin{array}{cc}
\cosh \left(\frac{1}{2} \hat{\theta}\right) & -r \sinh \left(\frac{1}{2} \hat{\theta}\right) \\
-r^{\dagger} \sinh \left(\frac{1}{2} \hat{\theta}\right) & \cosh \left(\frac{1}{2} \hat{\theta}\right)
\end{array}\right) \\
& =\operatorname{Sdet}^{-1 / 2}\left(\cosh ^{2}\left(\frac{1}{2} \hat{\theta}\right)-\sinh ^{2}\left(\frac{1}{2} \hat{\theta}\right) r^{\dagger} r\right) \\
& =\operatorname{Sdet}^{-1 / 2}\left(1+\sinh ^{2}\left(\frac{1}{2} \hat{\theta}\right) t^{\dagger} t\right),
\end{aligned}
$$

where we have again used (B1) and the relation $r^{\dagger} r=1-t^{\dagger} t$ imposed by unitarity of the scattering matrix.

The matrix $t^{\dagger} t$ has eigenvalues $T_{n}=\left(1+\lambda_{n}\right)^{-1}\left(n=1, \ldots, N_{1}\right)$, which are twofold degenerate for $\beta=4$, hence

$$
\begin{aligned}
F & =\prod_{n=1}^{N_{1}} \operatorname{Sdet}^{-d / 2}\left(1+\sinh ^{2}\left(\frac{1}{2} \hat{\theta}\right) T_{n}\right) \\
& =\prod_{n=1}^{N_{1}} \operatorname{Sdet}^{-d / 2}\left(\lambda_{n}+\cosh ^{2}\left(\frac{1}{2} \hat{\theta}\right)\right) .
\end{aligned}
$$

Here $d=1+\delta_{\beta, 4}$. Eq. (B6) establishes the connection between the two expressions (2.3) and (3.5) for the generating function.

The above calculation assumes $\mathcal{H}=H 1_{8}$, hence $\beta=1$ or $\beta=4$. In the case $\beta=2$, one has instead $\mathcal{H}=\operatorname{Re}(H) 1_{8}+i \operatorname{Im}(H) \tau_{3}$. Instead of two subblocks with $r$ and $r^{\dagger}$ [see Eq. (B3)], one now needs four subblocks with $r, r^{T}, r^{\dagger}$, and $r^{*}$. Repeating the calculation one finds that the final result (B6) remains the same.

\section{APPENDIX C: IDENTITY OF LAPLACIANS}

The goal of this appendix is to prove Eq. (2.6). Hereto we first analyze the structure of the 1.h.s. of Eq. (2.6) in more detail.

The derivatives of $F(\hat{\theta}, \hat{\lambda})$ with respect to $\theta_{j}$ are calculated using

$$
\begin{aligned}
\frac{\partial F(\hat{\theta}, \hat{\lambda})}{\partial \theta_{j}}= & \left(\sum_{n=1}^{N} \frac{1}{f\left(\hat{\theta}, \lambda_{n}\right)} \frac{\partial f\left(\hat{\theta}, \lambda_{n}\right)}{\partial \theta_{j}}\right) F(\hat{\theta}, \hat{\lambda}), \\
\frac{\partial^{2} F(\hat{\theta}, \hat{\lambda})}{\partial \theta_{j}^{2}}= & \left(\sum_{n=1}^{N} \frac{1}{f\left(\hat{\theta}, \lambda_{n}\right)} \frac{\partial^{2} f\left(\hat{\theta}, \lambda_{n}\right)}{\partial \theta_{j}^{2}}\right) F(\hat{\theta}, \hat{\lambda})+ \\
& \left(\sum_{n \neq m}^{N} \frac{1}{f\left(\hat{\theta}, \lambda_{n}\right) f\left(\hat{\theta}, \lambda_{m}\right)} \frac{\partial f\left(\hat{\theta}, \lambda_{n}\right)}{\partial \theta_{j}} \frac{\partial f\left(\hat{\theta}, \lambda_{m}\right)}{\partial \theta_{j}}\right) F(\hat{\theta}, \hat{\lambda}) .
\end{aligned}
$$


Inspection of Eqs. (2.4b), (C1) and (C2) shows that $\Delta_{\hat{\theta}} F(\hat{\theta}, \hat{\lambda})$ has two contributions, one involving a single summation over the channel indices $n$, and another one involving a double summation over channel indices $n \neq m$,

$$
\Delta_{\hat{\theta}} F(\hat{\theta}, \hat{\lambda})=\left(\sum_{n=1}^{N} g_{1}\left(\hat{\theta}, \lambda_{n}\right)\right) F(\hat{\theta}, \hat{\lambda})+\left(\sum_{n \neq m}^{N} g_{2}\left(\hat{\theta}, \lambda_{n}, \lambda_{m}\right)\right) F(\hat{\theta}, \hat{\lambda}) .
$$

Using the definition (2.3b) of $f(\hat{\theta}, \hat{\lambda})$ one may straightforwardly calculate the functions $g_{1}$ and $g_{2}$. The expressions are rather lengthy and will not be given here.

The r.h.s. of Eq. (2.6) contains the differential operator $D_{\hat{\lambda}}$, which is given by Eq. (2.5b). Simple algebra yields

$$
\begin{aligned}
D_{\hat{\lambda}}= & \sum_{n=1}^{N}\left(\lambda_{n}\left(1+\lambda_{n}\right) \frac{\partial^{2}}{\partial \lambda_{n}^{2}}+\left(1+2 \lambda_{n}\right) \frac{\partial}{\partial \lambda_{n}}\right)+ \\
& \frac{\beta}{2} \sum_{n \neq m}^{N}\left(\lambda_{n}-\lambda_{m}\right)^{-1}\left(\lambda_{n}\left(1+\lambda_{n}\right) \frac{\partial}{\partial \lambda_{n}}-\lambda_{m}\left(1+\lambda_{m}\right) \frac{\partial}{\partial \lambda_{m}}\right) .
\end{aligned}
$$

As a consequence, $D_{\hat{\lambda}} F(\hat{\theta}, \hat{\lambda})$ has again the structure of Eq. (C3), with $g_{1}$ and $g_{2}$ now given by

$$
\begin{gathered}
g_{1}(\hat{\theta}, \lambda)=\frac{1}{f(\hat{\theta}, \lambda)}\left[\lambda(1+\lambda) \frac{\partial^{2} f(\hat{\theta}, \lambda)}{\partial \lambda^{2}}+(1+2 \lambda) \frac{\partial f(\hat{\theta}, \lambda)}{\partial \lambda}\right], \\
g_{2}\left(\hat{\theta}, \lambda_{1}, \lambda_{2}\right)=\frac{\beta / 2}{\lambda_{1}-\lambda_{2}}\left[\frac{\lambda_{1}\left(1+\lambda_{1}\right)}{f\left(\hat{\theta}, \lambda_{1}\right)} \frac{\partial f\left(\hat{\theta}, \lambda_{1}\right)}{\partial \lambda_{1}}-\frac{\lambda_{2}\left(1+\lambda_{2}\right)}{f\left(\hat{\theta}, \lambda_{2}\right)} \frac{\partial f\left(\hat{\theta}, \lambda_{2}\right)}{\partial \lambda_{2}}\right] .
\end{gathered}
$$

Comparison of Eqs. (C3) and (C5) shows that the two definitions of the functions $g_{1}$ and $g_{2}$ are identical. This completes the proof of Eq. (2.6).

\section{APPENDIX D: EXTENSION TO HIGHER DIMENSIONAL SUPERMATRICES}

The argumentation presented in Secs. III and IV can be generalized to $\sigma$ models with $Q$ matrices of arbitrary dimension $8 p$ with $p \geq 1$. This generalized equivalence proof applies to the $p$-point functions $\rho_{p}\left(T_{1}, \ldots, T_{p}\right)$ instead to the limited number of statistical quantities that can be generated by the "small" $\sigma$ model with $p=1$ (compare appendix A). Here, we briefly present the modifications with respect to the $p=1$ case. The modifications concern the parameterization (2.1) and the generating function (2.3b).

The main technical difficulty in such a generalization is due to the radial part of the Laplace operator. The procedure to calculate it on conventional symmetric spaces is standard 30 and is carried over to the supersymmetric $\sigma$ models as described in appendix $\mathrm{B}$ of Ref. 11. It is now more convenient to use a slightly modified form of the parameterization of the $Q$-matrices, where $\hat{\theta}$ in Eq. (2.1a) is fully diagonal (rather than block-diagonal):

$$
\begin{aligned}
& \hat{\theta}=\left(\begin{array}{cc}
\hat{x} & 0 \\
0 & i \hat{y}
\end{array}\right), \\
& (\hat{x})_{n m}=x_{n} \delta_{n m},(\hat{y})_{n m}=y_{n} \delta_{n m}, 1 \leq n, m \leq 2 p .
\end{aligned}
$$


The symmetry restrictions are [cf. Eq. (2.1d)]

$$
\begin{aligned}
& y_{i}=y_{i+p} \quad \text { if } \beta=1 \text {, } \\
& x_{i}=x_{i+p}, y_{i}=y_{i+p} \text { if } \beta=2 \text {, } \\
& x_{i}=x_{i+p} \quad \text { if } \beta=4 \text {, }
\end{aligned}
$$

for $i=1, \ldots, p$. In the case $p=1$, we have the relations $x_{1}=\theta_{1}+\theta_{2}, x_{2}=\theta_{1}-\theta_{2}$, $y_{1}=\theta_{3}+\theta_{4}$, and $y_{2}=\theta_{3}-\theta_{4}$ between these parameters and the $\theta_{i}$ used in (2.1d).

We can directly apply the results of appendix B in Ref. 11 which are given in terms of the so-called roots $\alpha(\Theta)$ [with $\Theta$ given as in $(\overline{\mathrm{B} 4})$ ]. The roots are linear functions of $\Theta$ which are the eigenvalues of the linear mapping $\operatorname{ad}(\Theta)\left(X_{\alpha}\right):=\left[\Theta, X_{\alpha}\right]=\alpha(\Theta) X_{\alpha}$, defined on a certain super-Lie algebra.11 The eigenvectors $X_{\alpha}$ of the mapping are the root vectors, which do not depend on $\Theta$. The radial integration measure $J(\hat{\theta})$ in Eq. (2.4b) can be expressed as 30

$$
J(\hat{\theta})=\prod_{\alpha>0} \sinh ^{m_{\alpha}}\left[\frac{1}{2} \alpha(\Theta)\right]
$$

where the integer $m_{\alpha}$ is the multiplicity of the root $\alpha$ (the dimension of the root space). Both positive and negative values of $m_{\alpha}$ can occur. The factor $\frac{1}{2}$ is due to the difference between the normalization (2.1a) of $\Theta$ and the one used in Ref. 11. In appendix A of Ref. 11, explicit formulas for the roots as well as for the root vectors are given for the case $\beta=1$, $p=1$.

We have calculated the roots and the root vectors for all $\beta$ and arbitrary dimension $8 p$ of the $Q$ matrices. For simplicity, we only present the results for the roots and their multiplicities. Let us denote with $p_{x}\left(p_{y}\right)$ the number of independent $x_{i}\left(y_{i}\right)$ parameters, i.e.: $p_{x}=2 p, p, p$ and $p_{y}=p, p, 2 p$ for $\beta=1,2,4$, respectively. Note that $\beta p_{x}=2 p_{y}$. We find 8 different types of (positive) roots:

$$
\begin{array}{lll}
\alpha(\Theta)=x_{j}-x_{l} & \left(1 \leq j<l \leq p_{x}\right), & m_{\alpha}=\beta, \\
\alpha(\Theta)=x_{j}-i y_{l} & \left(1 \leq j \leq p_{x}, 1 \leq l \leq p_{y}\right), & m_{\alpha}=-2, \\
\alpha(\Theta)=i\left(y_{j}-y_{l}\right) & \left(1 \leq j<l \leq p_{y}\right), & m_{\alpha}=4 / \beta \\
\alpha(\Theta)=2 x_{j} & \left(1 \leq j \leq p_{x}\right), & m_{\alpha}=\beta-1 \\
\alpha(\Theta)=2 i y_{j} & \left(1 \leq j \leq p_{y}\right), & m_{\alpha}=4 / \beta-1, \\
\alpha(\Theta)=x_{j}+x_{l} & \left(1 \leq j<l \leq p_{x}\right), & m_{\alpha}=\beta, \\
\alpha(\Theta)=x_{j}+i y_{l} & \left(1 \leq j \leq p_{x}, 1 \leq l \leq p_{y}\right), & m_{\alpha}=-2, \\
\alpha(\Theta)=i\left(y_{j}+y_{l}\right) & \left(1 \leq j<l \leq p_{y}\right), & m_{\alpha}=4 / \beta .
\end{array}
$$

The radial part of the Laplacian takes the form

$$
\Delta_{\hat{\theta}}=\sum_{j=1}^{p_{x}} J^{-1}(\hat{\theta}) \frac{\partial}{\partial x_{j}} J(\hat{\theta}) \frac{\partial}{\partial x_{j}}+\frac{\beta}{2} \sum_{j=1}^{p_{y}} J^{-1}(\hat{\theta}) \frac{\partial}{\partial y_{j}} J(\hat{\theta}) \frac{\partial}{\partial y_{j}} .
$$

The expressions (2.3a) and (3.5a) for the generating function now remain valid with the modified $\hat{\theta}$ of Eq. (D1), and with Eq. (2.3b) replaced by

$$
f(\hat{\theta}, \lambda)=\prod_{i=1}^{p_{y}}\left[1+2 \lambda+\cos \left(y_{i}\right)\right] \prod_{i=1}^{p_{x}}\left[1+2 \lambda+\cosh \left(x_{i}\right)\right]^{-\beta / 2} .
$$


It is convenient to use the variables $u_{i}=\sinh ^{2}\left(\frac{1}{2} x_{i}\right)$ and $v_{i}=-\sin ^{2}\left(\frac{1}{2} y_{i}\right)$ in terms of which the Laplacian has the form

$$
\begin{aligned}
\Delta_{\hat{\theta}}= & \sum_{j=1}^{p_{x}} \tilde{J}^{-1} \frac{\partial}{\partial u_{j}} u_{j}\left(1+u_{j}\right) \tilde{J} \frac{\partial}{\partial u_{j}}-\frac{\beta}{2} \sum_{j=1}^{p_{y}} \tilde{J}^{-1} \frac{\partial}{\partial v_{j}} v_{j}\left(1+v_{j}\right) \tilde{J} \frac{\partial}{\partial v_{j}} \\
\tilde{J}= & \prod_{1 \leq i<j \leq p_{x}}\left(u_{i}-u_{j}\right)^{\beta} \prod_{1 \leq i<j \leq p_{y}}\left(v_{i}-v_{j}\right)^{4 / \beta} \prod_{i=1}^{p_{x}} \prod_{j=1}^{p_{y}}\left(u_{i}-v_{j}\right)^{-2} \\
& \times \prod_{i=1}^{p_{x}}\left(u_{i}\left(1+u_{i}\right)\right)^{\beta / 2-1} \prod_{i=1}^{p_{y}}\left(v_{i}\left(1+v_{i}\right)\right)^{2 / \beta-1} .
\end{aligned}
$$

The generating function $F(\hat{\theta}, \hat{\lambda})$ is given by

$$
F(\hat{\theta}, \hat{\lambda})=\prod_{n=1}^{N}\left(\prod_{i=1}^{p_{y}}\left(1+\lambda_{n}+v_{i}\right) \prod_{i=1}^{p_{x}}\left(1+\lambda_{n}+u_{i}\right)^{-\beta / 2}\right) .
$$

We have verified that the identity of Laplacians [Eq. 2.6)] remains true for the modified expressions (D7a) and (D8). The calculations goes in a similar way as shown in App. 9 for $p=1$. Now, we have to keep track of 7 different types of contributions with double and triple sums over functions of $\lambda_{n}, u_{i}, v_{j}$.

In App. A we have shown that the average density of transmission eigenvalues $\rho(T)$ can be obtained from the generating function (2.3a). Using the corresponding function for the higher-dimensional $\sigma$ model considered here, it is straightforward to get the $p$-point correlation functions $\rho_{p}\left(T_{1}, \ldots, T_{p}\right)$. 


\section{REFERENCES}

${ }^{1}$ O. N. Dorokhov, Pis'ma Zh. Eksp. Teor. Fiz. 36, 259 (1982) [JETP Lett. 36, 318 (1982)].

${ }^{2}$ P. A. Mello, P. Pereyra, and N. Kumar, Ann. Phys. (NY) 181, 290 (1988).

${ }^{3}$ P. A. Mello, Phys. Rev. Lett. 60, 1089 (1988).

${ }^{4}$ P. A. Mello and A. D. Stone, Phys. Rev. B 44, 3559 (1991).

${ }^{5}$ A. M. S. Macêdo and J. T. Chalker, Phys. Rev. B 46, 14985 (1992).

${ }^{6}$ K. B. Efetov and A. I. Larkin, Zh. Eksp. Theor. Fiz. 85, 764 (1983) [Sov. Phys. JETP 58, $444(1983)]$.

${ }^{7}$ K. B. Efetov, Adv. in Phys. 32, 53 (1983).

${ }^{8}$ J. J. M. Verbaarschot, H. A. Weidenmüller, and M. R. Zirnbauer, Phys. Rep. 129, 367 (1985).

${ }^{9}$ S. Iida, H. A. Weidenmüller, and J. A. Zuk, Ann. Phys. (NY) 200, 219 (1990).

${ }^{10}$ M. R. Zirnbauer, Phys. Rev. Lett. 69, 1584 (1992).

${ }^{11}$ A. D. Mirlin, A. Müller-Groeling, and M. R. Zirnbauer, Ann. Phys. (NY) 236, 325 (1994).

${ }^{12}$ C. W. J. Beenakker and B. Rejaei, Phys. Rev. Lett. 71, 3689 (1993); Phys. Rev. B 49, 7499 (1994).

${ }^{13}$ K. Frahm, Phys. Rev. Lett. 74, 4706 (1995); K. Frahm and J.-L. Pichard, J. Phys. I France 5, 877 (1995).

${ }^{14}$ M. Caselle, Phys. Rev. Lett. 74, 2776 (1995).

${ }^{15}$ B. Rejaei, Phys. Rev. B, to be published.

${ }^{16}$ A. Altland, Z. Phys. B 82, 105 (1991).

${ }^{17}$ P. W. Anderson, E. Abrahams, and T. V. Ramakrishnan, Phys. Rev. Lett. 43, 718 (1979).

${ }^{18}$ L. P. Gor'kov, A. I. Larkin, and D. E. Khmel'nitskiǐ, Pis'ma Zh. Eksp. Teor. Fiz. 30, 248 (1979) [JETP Lett. 30, 228 (1979)].

${ }^{19}$ B. L. Al'tshuler, Pis'ma Zh. Eksp. Teor. Fiz. 41, 530 (1985) [JETP Lett. 41, 648 (1985)].

${ }^{20}$ P. A. Lee and A. D. Stone, Phys. Rev. Lett. 55, 1622 (1985); P. A. Lee, A. D. Stone and H. Fukuyama, Phys. Rev. B 35, 1039 (1987).

${ }^{21}$ A. D. Mirlin and A. Müller-Groeling, unpublished calculations.

${ }^{22}$ P. A. Mello and B. Shapiro, Phys. Rev. B 37, 5860 (1988).

${ }^{23}$ P. A. Mello and S. Tomsovic, Phys. Rev. Lett. 67, 342 (1991); Phys. Rev. B 46, 15963 (1992).

${ }^{24}$ A. Hüffmann, J. Phys. A 23, 5733 (1990).

${ }^{25}$ F. J. Wegner, Phys. Rev. B 19, 783 (1979).

${ }^{26}$ Y. V. Fyodorov and A. D. Mirlin, Phys. Rev. Lett. 67, 2405 (1991).

${ }^{27}$ Yu. V. Nazarov, Phys. Rev. Lett. 73, 134 (1994).

${ }^{28}$ A. D. Stone, P. A. Mello, K. A. Muttalib, and J.-L. Pichard in Mesoscopic Phenomena in Solids, edited by B. L. Altshuler, P. A. Lee, and R. A. Webb (North Holland, Amsterdam, 1991).

${ }^{29}$ R. Landauer, Phil. Mag. 21, 863 (1970).

${ }^{30}$ S. Helgason, Groups and Geometric Analysis (Academic, Orlando, 1984). 


\section{FIGURES}

FIG. 1. Schematic drawing of the disordered wire and the leads according to the IWZ model description. The left lead (lead 1) contains $N_{1}$, the right lead (lead 2) $N_{2}$ propagating channels. The number of propagating channels in the disordered wire is $N$. In the IWZ model, the disordered wire is divided into $K$ segments, each having a random Hamiltonian drawn drom the Gaussian ensemble. The derivation of the $1 d \sigma$ model from the IWZ model assumes $N \gg 1$, but allows for finite $N_{1}$ and $N_{2}$.

FIG. 2. The average conductance $\langle g\rangle$ multiplied by $4 L / \xi=L / N \ell$ for the symplectic symmetry class as a function of $4 L / \xi$ for $N \gg 1$. Shown are our result (5.8) (solid), the numerical simulation of Ref. 21 ( $M=100, N=25)$ (dashed), and the result of Ref. 11 (dotted).

FIG. 3. As in Fig. 2 for the variance var $g$ of the conductance.

FIG. 4. The average conductance $\langle g\rangle$ multiplied by $\beta L / \xi=L / N \ell$ for the three symmetry classes as a function of $\beta L / \xi$ for $N \gg 1$. The curves for $\beta=1,2$ are taken from Refs. 10 and 11 and the curve for $\beta=4$ is calculated from Eq. (5.8). Notice that $\xi=\beta N \ell$ is proportional to $\beta$, so that the scaling of the axes is $\beta$-independent.

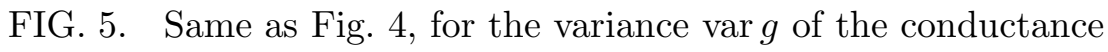




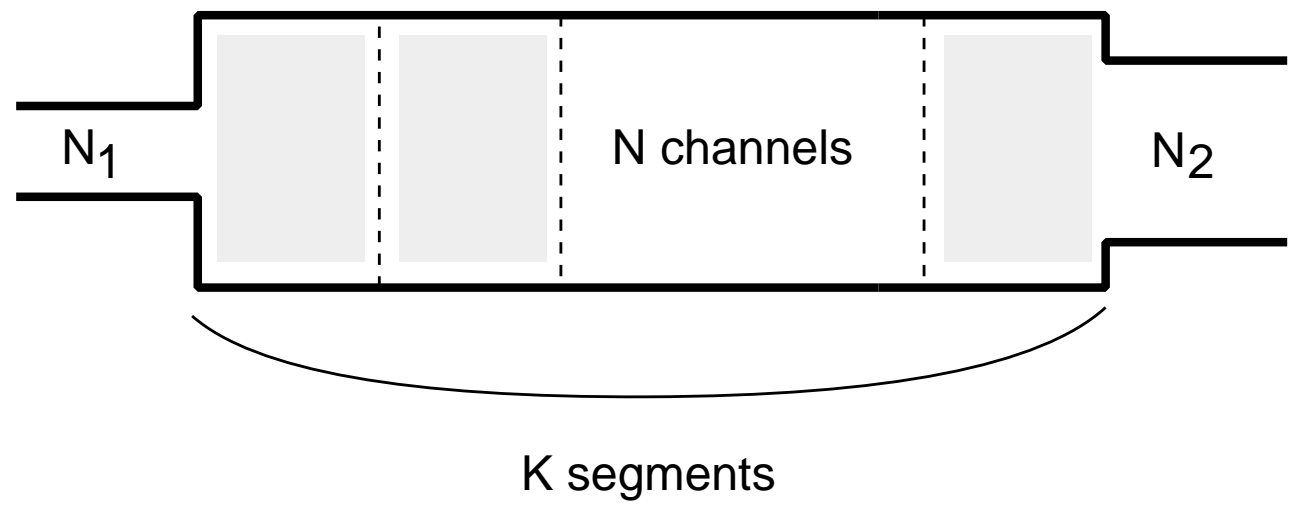

Fig. 1. 


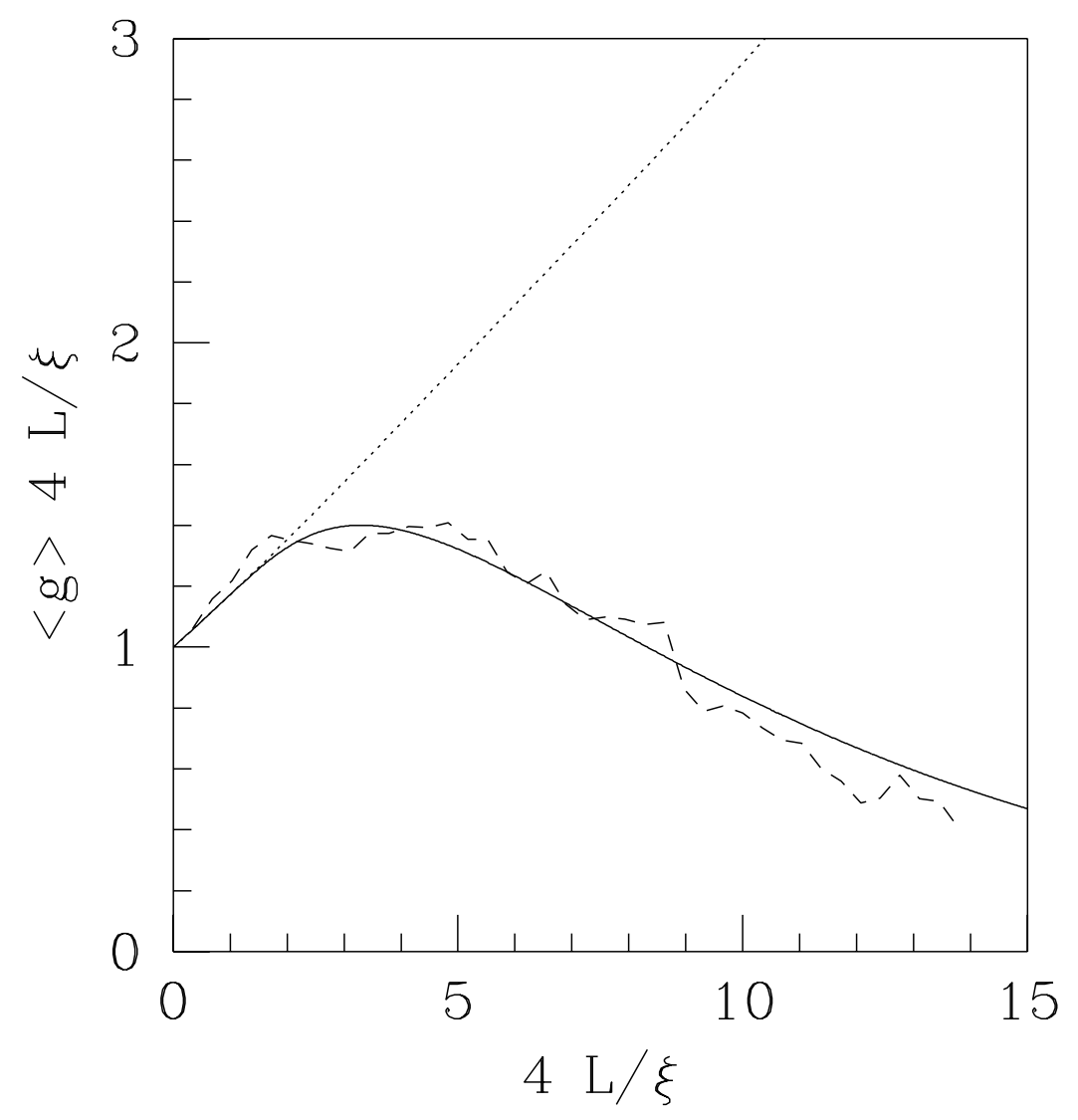

Fig. 2. 


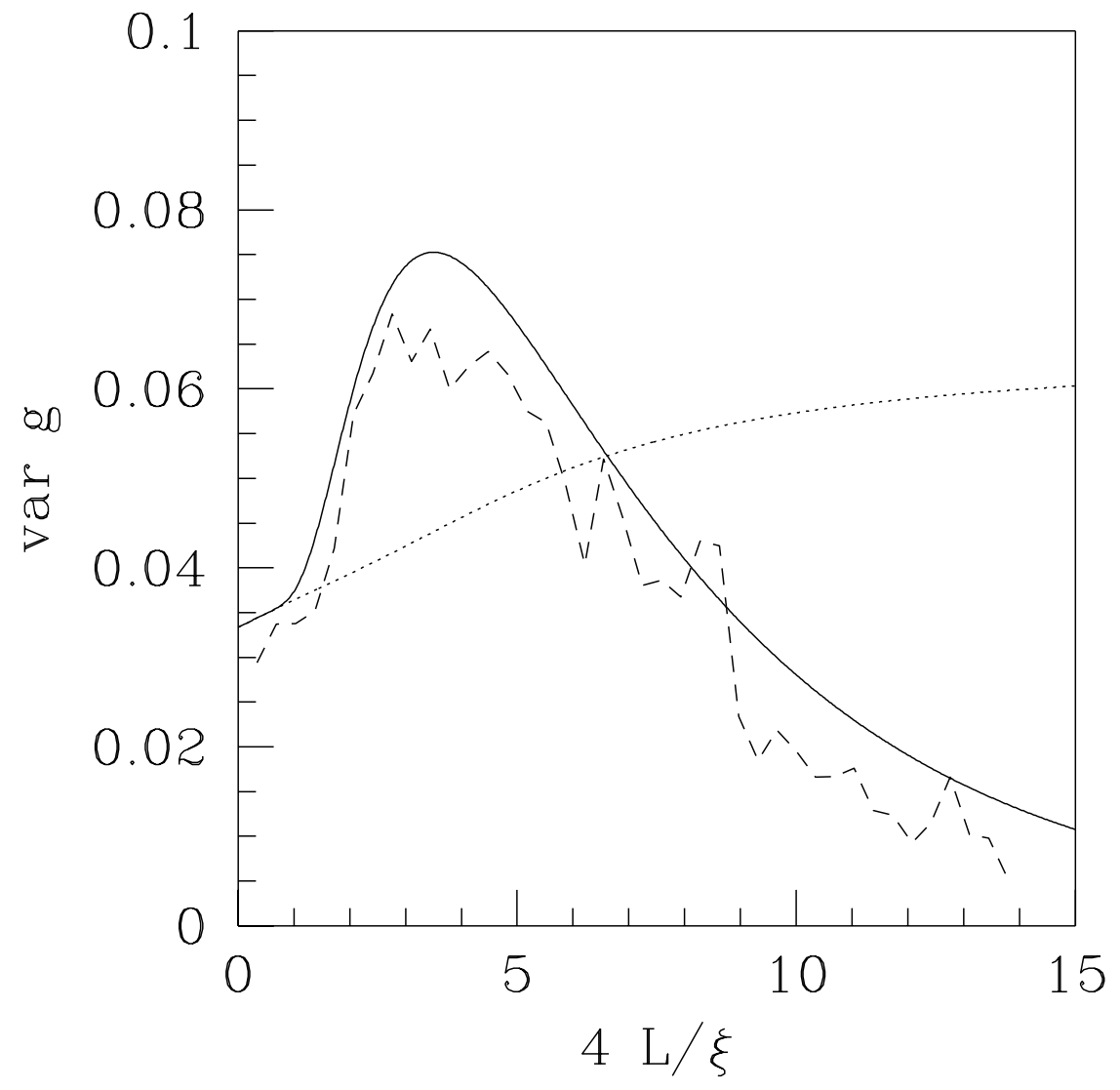

Fig. 3 


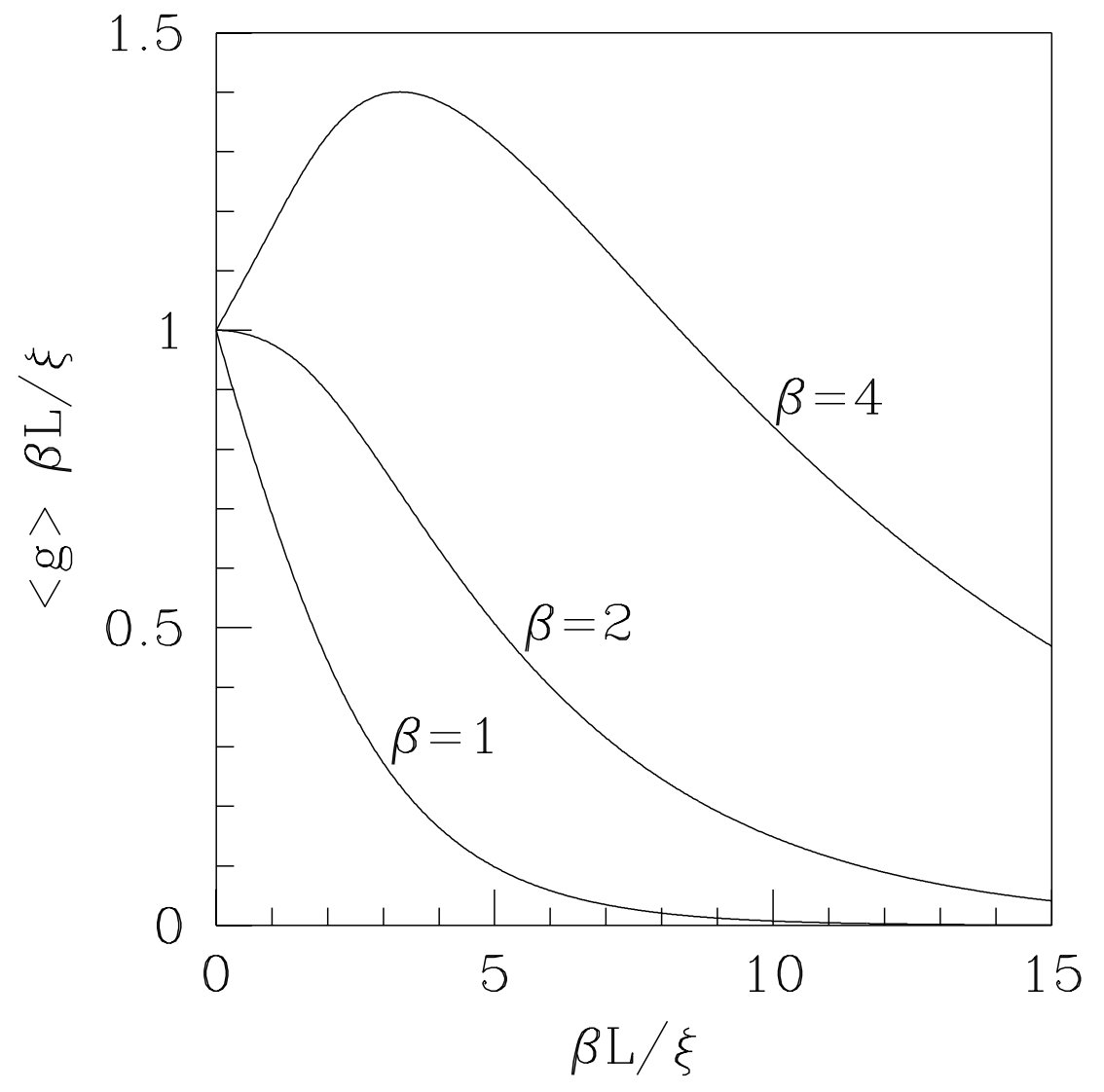

Fig. 4. 


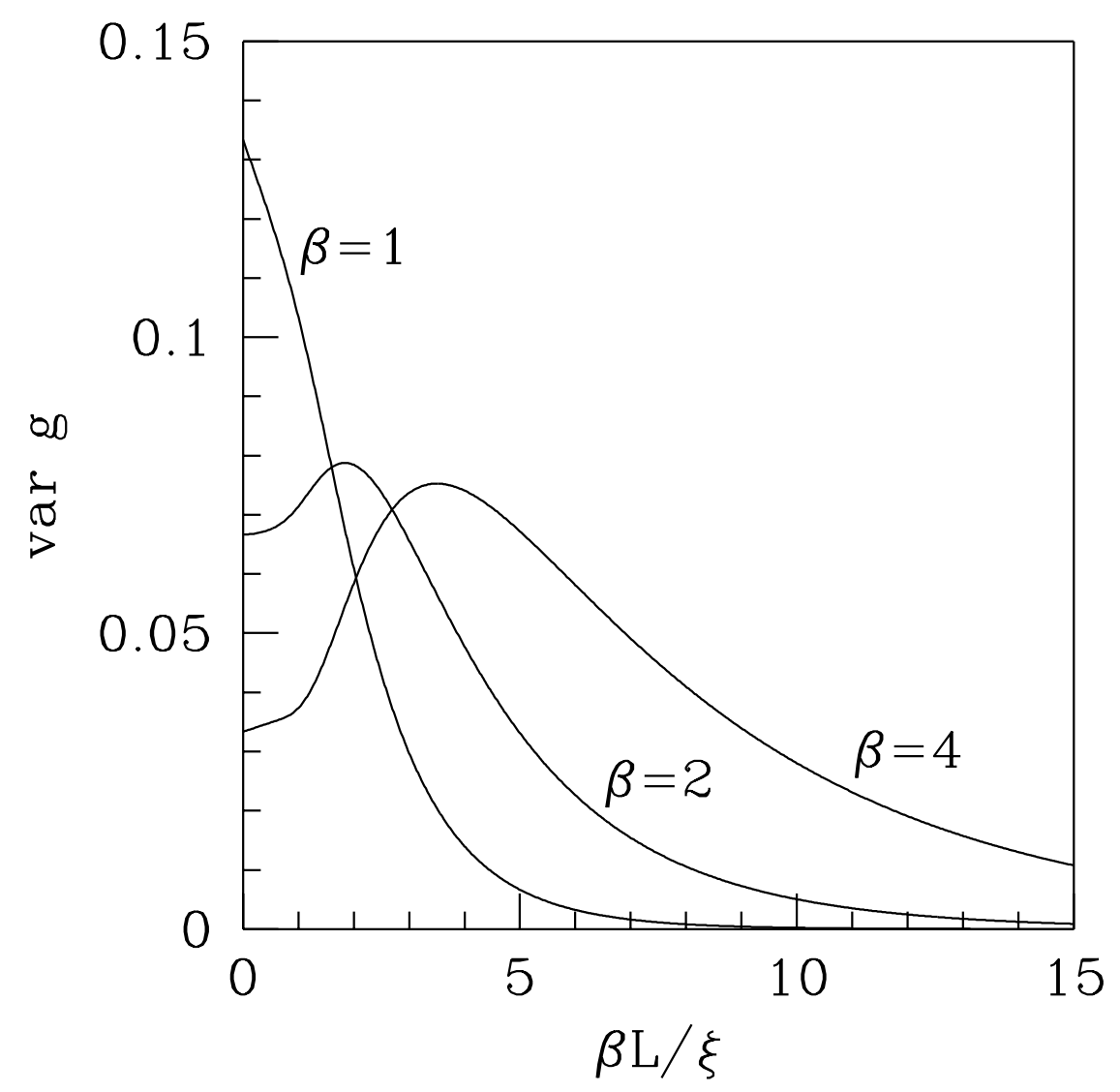

Fig. 5. 